\title{
Redundant Nerve Roots of Cauda Equina Mimicking Intradural Disc Herniation: A Case Report
}

\author{
Sang Mi Yang, Hyung Ki Park, Sung Jin Cho, Jae Chil Chang \\ Department of Neurosurgery, College of Medicine, Soonchunhyang University, Seoul, Korea
}

Redundant Nerve Roots (RNRs) is an uncommon clinical condition characterized by a tortuous, serpentine, large and elongated nerve root of the cauda equina. To our knowledge, most cases of RNRs are associated with lumbar stenosis, and RNRs associated with lumbar disc herniation has not been reported until now.

Here we present a rare case of unusual RNRs associated with lumbar disc herniation mimicking intradural disc herniation.

Key Words: Redundant nerve roots $\cdot$ Lumbar disc herniation

\section{INTRODUCTION}

RNRs of the cauda equina were first described by Verbiest ${ }^{14)}$ in 1954, and subsequently named by Cressman and Paul ${ }^{1)}$ in 1968. RNRs is characterized by a tortuous, serpentine, large and elongated nerve root of the cauda equina ${ }^{10)}$. It has been reported that RNRs develop as a response to lumbar spinal stenosis ${ }^{3,7,10)}$. To our knowledge, most RNRs are associated with lumbar spinal stenosis, and the RNRs associated with lumbar disc herniation has not been reported until now.

Therefore, we present a rare case of unusual RNRs associated with lumbar disc herniation mimicking intradural disc herniation on magnetic resonance imaging study.

\section{CASE REPORT}

A 50-year-old man who worked at a rice-cake shop has suffered from increasing low back pain and radiating pain on his left leg for five years. He had a nerve root block on L5 level five years ago, and the pain subsided temporarily. He was having conservative treatment but mild chronic pain still lasted and gradually aggravated and abruptly extended to the right leg a few days before his admission. Radiating pain was

\footnotetext{
- Received: May 7, 2012 - Revised: February 3, 2013

- Accepted: March 22, 2013

Corresponding Author: Jae Chil Chang, MD

Department of Neurosurgery, Soonchunhyang University Hospital 657

Hannam-dong, Yongsan-Gu, Seoul 140-743, Korea

Tel: +82-2-709-9268, Fax: +82-2-792-5976

E-mail: j7chang@gmail.com
}

on posterior and lateral aspect of the leg and toe.

He had no significant medical history or trauma history of spine. But he has been lifting heavy things repeatedly at work. A neurological examination revealed no paresthesia or motor weakness. A straight leg raise test was positive on the left side and the bilateral knee and ankle jerk was normal. Micturition and defecation were not disturbed.

Sagittal and axial T2-weighted magnetic resonance image reveals broad base central disc protrusion at L4-5 level, and about $0.7 \times 0.5 \times 2.5 \mathrm{~cm}$ sized ovoid nonenhancing lesion in dorsal aspect of L5-S1, which seemed to be intradural disc herniation rather than intradural mass (Fig. 1). Although the image clearly demonstrated neither the classical ring enhancement of the intradural fragments on gadolinium contrast MRI or discontinuity of the PLL disrupted at the disc level, this finding was thought as an intradural disc herniation rather than an intradural extramedullary (IDEM) tumor such as meningioma. CT myelography showed central disc herniation at L4-5 and intradural lesion looked like intradural disc or intradural mass within the thecal sac below L4/5 (Fig. 2).

A decompressive laminectomy was carried out at L4-5 levels and a herniated disc at L4-5 was removed. And we found the fragments of ruptured disc and adhesion with the dura. To determine whether there was a coexisting intradural disc rupture, we carried out an intradural exploration. After durotomy, a long, edematous and tortuous nerve root had forced out through a dural incision (Fig. 3). We explored the thecal sac, but there was no other intradural abnormality such as intradural disc herniation or intradural mass.

After rearrangement of RNRs of the cauda equina to be released from the tangled position, the dura was carefully closed without tension. 

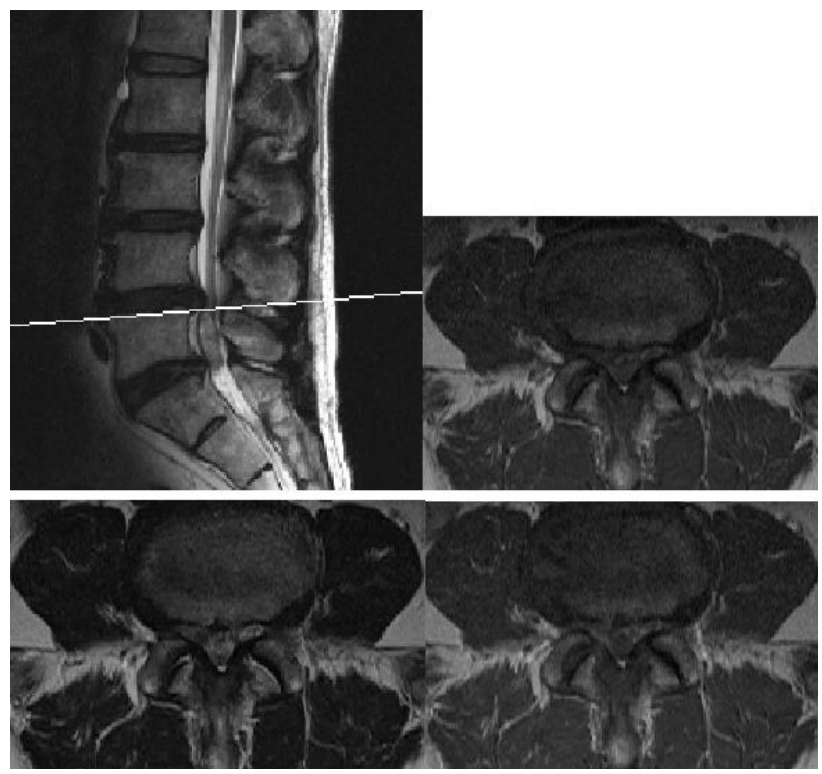

Fig. 1. Sagittal and axial T2-weighted magnetic resonance image reveals broad base central disc protrusion at the L4-5 level, and about $0.7 \times 0.5 \times 2.5 \mathrm{~cm}$ sized ovoid nonenhancing lesion in dorsal aspect of L5-S1, which seems probably intradural disc herniation rather than intradural mass.

The radiating pain was improved immediately in the postoperative period. The patient made an uneventful recovery and returned to normal activities. One year after surgery, he had no further pain.

\section{DISCUSSION}

The RNRs of the cauda equina has been discussed sporadically in both the neurological and radiological literature. Characteristically, the patients are men in their $4^{\text {th }}$ to $6^{\text {th }}$ decade, and the L3 to S1 nerve roots are most commonly involved ${ }^{4)}$.

RNRs are relatively common finding in association with spinal stenosis ${ }^{5}$. RNRs were found in $42.3 \%$ of the patients with severe dural constriction and were seen on $8.2 \%$ of cadavers of aged people in autopsy ${ }^{10)}$. Low back pain, lumbar radiculopathy, and intermittent claudication are common presenting features ${ }^{3)}$. Associated bladder and sexual dysfunction can also occur ${ }^{3,6}$. The disorder is usually found in older patients and the onset of symptoms exhibits a long period ${ }^{10)}$.

The cause of RNRs is unknown and is a matter of controversy. It was once presumed to be a rare congenital anomaly. Patients with achondroplasia may have $\mathrm{RNR}^{8}{ }^{8}$. Some authors suggested that congenitally long nerve roots in a narrow canal create a block ${ }^{1,8)}$. There are a few cases associated with the presence of an arachnoid cyst ${ }^{9,11)}$. There are many reports on the relationship between RNRs and constriction of the spinal

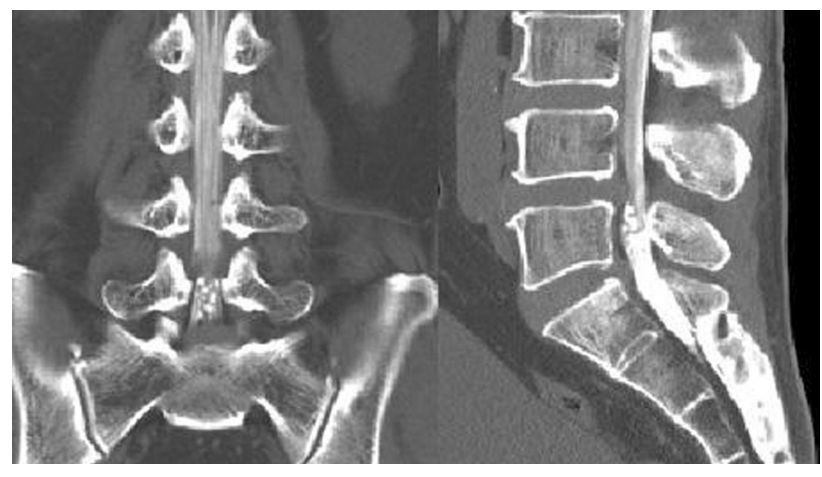

Fig. 2. Preoperative CT myelography shows central disc herniation at L4-5 and intradural lesion looked like intradural disc or intradural mass within the thecal sac below L4/5.

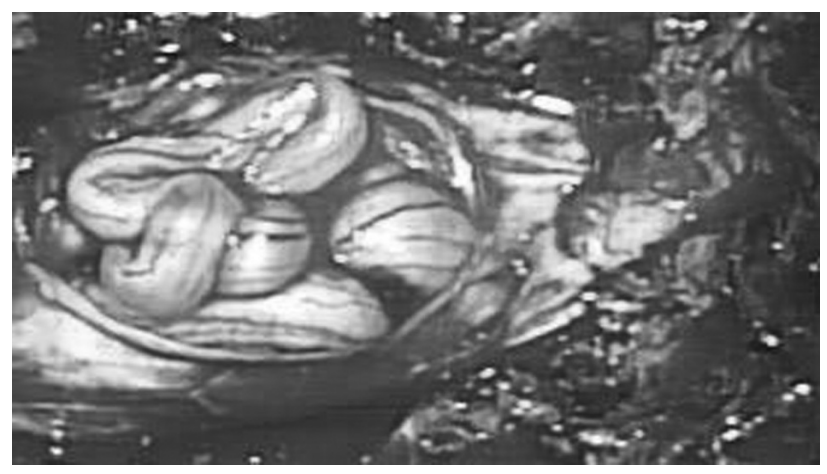

Fig. 3. Intraoperative dorsal view of the elongated, edematous nerve root of the cauda equina. A non-pulsatile dural sac suddenly extruded through the dural incision as if it was squeezed from a tube of toothpaste.

canal and the most substantial mechanism seems to be an acquired elongation of nerve roots due to mechanical trapping at the site of spinal stenosis ${ }^{2,3,4,10,13,14)}$. The inhibition of normal sliding and mobility causes repeated stretching of the nerves, leading to elongation above the level of constriction. When positional changes (e.g., flexion) cause a cephalic pull on the nerve roots, they are caught at the constriction and are unable to slide through. This repeated stretch causes elongation of the roots above the block. When the nerve roots are relieved, the elongated segments relax and pile up in redundant loops above the block ${ }^{2}$. Different from many cases of RNRs presenting above the stenosis, redundant nerve roots were below L4/5 level that had a broad base central disc protrusion in our case. The pathogenic mechanism seems to be different from the RNRs associated with spinal stenosis. We suppose that disc protrusion had an effect on the thecal sac and caused the RNRs. But, it is still a question why the RNRs developed below the block, and not above, and there is lack of sufficient analysis about the RNRs developed below the block in the previous study. 
The importance of recognizing RNRs is to avoid misdiagnosis. In previous reports of RNRs, the evaluations were mainly based on myelographic findings. Though the appearance of serpiginous filling defects can be helpful in differentiating from other causes, it can also cause confusion occasionally, so it must be differentiated from vascular lesions such as arteriovenous malformation or dilated tortuous veins due to other causes ${ }^{2,3)}$. Arteriovenous malformations can also produce serpiginous filling defects, but usually are not associated with extradural blocks. They, usually thoracic in location, show no variability with positional change and often have large feeding vessels ${ }^{2,9)}$. Venous engorgement resulting from impaired venous drainage secondary to a block produces filling defects below the block. Also, the positional changes seen with redundant roots should not occur ${ }^{2,6,12)}$.

RNRs could also be diagnosed on sagittal T2-weighted MR images. In general, the positive rate of RNRs examined by the MR imaging was as high as $97.6 \%$ when the nerve roots appeared as loop-shaped lesion on myelograms, whereas the positive rate was only $23.5 \%$ when the nerve roots appeared as serpentine-shaped lesions. According to the previous study, Magnetic resonance imaging appeared to demonstrate more advanced RNRs, and therefore MR imaging features of the RNRs are thought to represent important clinical findings ${ }^{7}$. Unfortunately, MR imaging of the present case didn't show definite features of usual RNRs in spinal stenosis and it was not enough to exclude intradural herniation or intradural mass. Untypically, the RNRs looked like intradural mass such as IDEM tumor, but the lesion was not enhanced unlike intradural mass. The RNRs had similar signal intensity to the cauda equina on both T1 and T2-weighted images but on enhanced images, it is observed as a non-enhancing lesion protruding on intradural space of L4-5. In addition, there was disc protrusion on L4/5 accompanied by non-enhanced intradural lesion just below L4/5.

Also, CT myelography showed central disc herniation at L4/5 and intradural lesion looking like intradural disc or intradural mass within the thecal sac below L4/5. It is possible to mistake the intradural lesion as the downward migrating intradural ruptured disc, so we performed the intradural exploration to confirm. To avoid misdiagnosis and unnecessary procedure, careful analysis and wide experiences are needed.

Early and sufficient decompressive laminectomy with duroplasty is recommended to relieve the nerve root compression before neurological deficits appear ${ }^{3,6}$. Relief of RNRs at an early stage is strongly recommended. Patients with severe dural constriction caused by lumbar spinal stenosis should be treated surgically before the nerve roots undergo irreversible damage ${ }^{10)}$ and intradural exploration should be considered where there still exists a question of tumor or AVM.

\section{CONCLUSION}

We experienced untypical RNRs and the RNRs with lumbar disc herniation could have been misdiagnosed as the intradural disc herniation before operation. To avoid misdiagnosis, it needs to be notice that lumbar disc herniation could be causative of RNRs. So we report a rare case of RNRs associated with lumbar disc herniation mimicking intradural disc herniation.

\section{REFERENCES}

1. Cressman MR, Pawl RP: Serpentine myelographic defect caused by a redundant nerve root. case report. J Neurosurg 28:391393, 1968

2. Hacker DA, Latchaw RE, Yock DH Jr, Ghosharjura K, Gold LH: Redundant lumbar nerve root syndrome: myelographic features. Radiology 143:457-461, 1982

3. Hakan T, Celikoglu E, Aydoseli A, Demir K: The redundant nerve root syndrome of the cauda equina. Turk Neurosurg 18: 204-206, 2008

4. Lee SH, Park SH, Hamm IS, Park YM: A case of the redundant nerve root syndrome mimicking intradural spinal tumor. J Korean Neurosurg Soc 36:160-162, 2004

5. Min JH, Jang JS, Lee SH: Clinical significance of redundant nerve roots of the cauda equina in lumbar spinal stenosis. Clin Neurol Neurosurg 110:14-18, 2008

6. Naguib MG, Latchaw RE, Erickson DL, Seljeskog EL: Redundant nerve roots of the cauda equina. Neurosurgery 9:444-449, 1981

7. Ono A, Suetsuna F, Irie T, Yokoyama T, Numasawa T, Wada K, et al: Clinical significance of the redundant nerve roots of the cauda equina documented on magnetic resonance imaging. J Neurosurg Spine 7:27-32, 2007

8. Pau A, Turtas S: Redundant nerve roots of the cauda equina. a case report. Acta Neurochir(Wien) 33:311-317, 1976

9. Rigsby CM, Virapongse C, Duncan C: Positional variability in redundant lumbar nerve-root syndrome. Surg Neurol 19:513516, 1983

10. Suzuki K, Ishida Y, Ohmori K, Sakai H, Hashizume Y: Redundant nerve roots of the cauda equina: clinical aspects and consideration of pathogenesis. Neurosurgery 24:521-528, 1989

11. SWV Chan RL, MT Chau: Redundant nerve roots of the cauda equina associated with lumbar spinal arachnoid cyst. Hong Kong J Radiol 14:113-117, 2011

12. Thulin CA, Edner G, Fodstad H, Salford L, Svendgaard NA: Redundant nerve roots of the cauda equina. a report of five cases. Acta Neurochir (Wien) 41:115-125, 1978

13. Tsuji H, Tamaki T, Itoh T, Yamada H, Motoe T, Tatezaki S, et al: Redundant nerve roots in patients with degenerative lumbar spinal stenosis. Spine (Phila Pa 1976) 10:72-82, 1985

14. Verbiest $\mathrm{H}$ : A radicular syndrome from developmental narrowing of the lumbar vertebral canal. J Bone Joint Surg Br 36-B: 230-237, 1954 